\title{
Loss of Transfected Human Brain Micro-Vascular Endothelial Cell Integrity during Herpes Simplex Virus Infection
}

\author{
Sau Har Lee ${ }^{a, b}$ Nadia Atiya ${ }^{b}$ Seok Mui Wang ${ }^{d}$ Rishya Manikam ${ }^{c}$ \\ Chandramathi Samudi Raju ${ }^{b}$ Shamala Devi Sekaran ${ }^{\mathrm{e}}$ \\ a School of Biosciences, Faculty of Health and Medical Sciences, Taylor's University, Subang Jaya, Malaysia; \\ ${ }^{b}$ Department of Medical Microbiology, Faculty of Medicine, University of Malaya, Kuala Lumpur, Malaysia; \\ 'Department of Trauma and Emergency Medicine, University of Malaya Medical Centre, Kuala Lumpur, Malaysia; \\ dFaculty of Medicine, Sungai Buloh Campus, Universiti Teknologi MARA, Sungai Buloh, Malaysia; eFaculty of \\ Medicine and Biomedical Sciences, MAHSA University, Saujana Putra Campus, Jenjarum, Malaysia
}

\section{Keywords}

Viral encephalitis · Herpes simplex virus · Blood brain barrier · Electrical cell-substrate impedance sensing ·

Resistance · Capacitance

\begin{abstract}
Objective: Herpes simplex virus infection through the neuronal route is the most well-studied mode of viral encephalitis that can persists in a human host for a lifetime. However, the involvement of other possible infection mechanisms by the virus remains underexplored. Therefore, this study aims to determine the temporal effects and mechanisms by which the virus breaches the human brain micro-vascular endothelial cells of the blood-brain barrier. Method: An electrical cellsubstrate impedance-sensing tool was utilized to study the real-time cell-cell barrier or morphological changes in response to the virus infection. Results: Herpes simplex virus, regardless of type (i.e., 1 or 2), reduced the cell-cell barrier resistance almost immediately after virus addition to endo-
\end{abstract}

thelial cells, with negligible involvement of cell-matrix adhesion changes. There is no exclusivity in the infection ability of endothelial cells. From $30 \mathrm{~h}$ after HSV infection, there was an increase in cell membrane capacitance with a subsequent loss of cell-matrix adhesion capability, indicating a viability loss of the infected endothelial cells. Conclusion: This study shows for the first time that destruction of human brain micro-vascular endothelial cells as an in vitro model of the blood-brain barrier could be an alternative invasion mechanism during herpes simplex virus infection.

(c) 2018 S. Karger AG, Basel

\section{Introduction}

Encephalitis or brain inflammation is a global neurological disorder that affects people of all ages, with a predominance in children. Despite the rarity of the disease ( 3.5-7.4 per 100,000 patient years), it has attracted public attention due to its high morbidity and mortality.

\section{KARGER}

(c) 2018 S. Karger AG, Basel

E-Mail karger@karger.com

www.karger.com/int
Shamala Devi Sekaran

Faculty of Medicine and Biomedical Sciences, MAHSA University

Saujana Putra Campus, Jalan SP2, Selangpr

Jenjarum, Selangor Darul Ehsan 42610 (Malaysia)

E-Mail shamalamy@yahoo.com 
Encephalitis is unavoidable as a common pathological response to brain infection, mainly involving viruses [1]. There are more than 11 virus families that could infect the brain, including DNA viruses, RNA viruses, and retroviruses [2]. Regardless of the virus type, breaching of the blood-brain barrier (BBB) is a common phenomenon [2]. The $B B B$ is supposed to be a protective layer that separates the cerebral tissue from the peripheral blood circulatory system. The primary component of this barrier includes a lining of endothelial cells that forms the wall of the brain capillaries. In addition to the lack of fenestration in these specialized brain endothelial cells, they also possess extraordinarily tight cell-cell junctions that enable only the passage of less than $2 \%$ of small molecules from the circulation into the central nervous system $[2,3]$. Although it could keep most of the pathogens away, the BBB does not seem to be able to withstand the assault of several virus types, including human immunodeficiency virus, West Nile virus and mouse adenovirus type 1 [2].

Herpes simplex virus (HSV), now known as human alphaherpesvirus 1, is a DNA virus from the Herpesviridae family with a $152-\mathrm{kbp}$ double-stranded DNA genome. Although HSV type 1 (HSV-1) and type 2 (HSV2 ) evolved in similar ways, they are 2 diverged species with only about $50 \%$ similarity in terms of their genetic homology $[4,5]$. Nevertheless, HSV-1 and HSV-2 are able to establish latency and present in the host for an entire life and cause secondary infections, which might lead to HSV encephalitis (HSVE). HSVE is a highly morbid acute disease usually accompanied by headache, fever, seizures, decreased consciousness, and focal neurological activity [6]. HSV is well known to invade the nervous system through a neuronal route by fusing and maintaining latency within the axon terminus of the neuronal receptor until it is reactivated to cause severe disease in the peripheral or central nervous system. However, it was unclear whether HSV could invade the brain directly by violating the protective $\mathrm{BBB}$, which is the entry mechanism utilized by several common DNA viruses such as JC virus, Epstein-Barr virus, and cytomegalovirus [7]. This mechanism is highly probable during HSV infection as a single virus type could act in diverse manners depending on the individual health condition and brain region involved [8]. Besides, the time course for HSV infection through brain micro-vascular endothelial cells (MEC), the major components of the $\mathrm{BBB}$, is not yet fully understood.

Electrical cell-substrate impedance sensing (ECIS) is an effective electrical system established in 1984 by Gi- aever and Keese, who used the system to replace microscopes for real-time measurement of cell behavior changes in an adherent monolayer, hence allowing studies of cell attachment, growth, movement, functions, and morphology alterations $[9,10]$. The development of this technology has become a valuable research tool to complement whole tissue and in vivo experiments for advancing our understanding of vascular and neurovascular diseases [9]. In our case, the use of this technology enabled us to monitor the changes in the BBB when it was exposed to HSV infection. Therefore, the purpose of this study is to determine the alteration mechanisms in endothelial barrier integrity during HSV infection and the time required for the virus to breach the protective barrier.

\section{Materials and Methods}

\section{In vitro MEC Model}

The in vitro MEC model used in this study is a transfected human brain MEC (THBMEC), a gift from Dr. Anna Durban of the John Hopkins University School of Medicine. This THBMEC cell line was stably immortalized with a pSVT plasmid that contains the DNA sequence encoding the SV40-large T antigen. It forms a polarized monolayer during confluence with characteristics similar to the primary human brain MEC. This human cell line is commonly used to study BBB function and permeability [11, 12]. THBMEC was propagated using M199 medium supplemented with $5 \%$ heat-inactivated fetal bovine serum (FBS) and $1 \%$ penicillin/streptomycin antibiotics. The cell was cultured in a humidified incubator maintained at $37^{\circ} \mathrm{C}$ with $5 \% \mathrm{CO}_{2}$.

\section{Virus Propagation}

Clinically isolated HSV-1 and HSV-2 were acquired from the Department of Medical Microbiology of the University of Malaya [13]. Both HSV-1 and HSV-2 were propagated in the African green monkey kidney (Vero cells, CCL-81), which was acquired from ATCC, USA. This cell line was used as a virus propagation medium since it supported the growth of numerous viruses. Vero cells were cultured in Dulbecco's modified Eagle medium (Hyclone, USA) supplemented with $5 \%$ heat-inactivated FBS and were kept in a humidified incubator maintained at $37^{\circ} \mathrm{C}$ with $5 \% \mathrm{CO}_{2}$.

For virus propagation, $50-100 \mu \mathrm{L}$ of the virus was inoculated onto $95 \%$ confluence Vero cells and incubated at $37^{\circ} \mathrm{C}$ with $5 \%$ $\mathrm{CO}_{2}$. The cells were then observed regularly for syncytia formation, which indicated a cytopathic feature $[14,15]$. As the virus replicates in the cells and the virus number increases, a higher number of syncytia or cytopathic effects can be observed. Once $90-95 \%$ of syncytia were observed on the cell monolayer, the culture supernatant containing virus was collected and centrifuged at $2,500 \mathrm{rpm}, 4^{\circ} \mathrm{C}$ for $10 \mathrm{~min}$ (centrifuge 5810R, rotor A-4-81; Eppendorf) to remove cell debris. These virus stocks were then aliquoted and stored at $-80^{\circ} \mathrm{C}$ for virus titration or until further use. At the same time, the culture supernatant of the non-HSV-infected Vero cells was also collected and aliquoted to be used as the control group for the subsequent experiments. 
Fig. 1. HSV-1 and HSV-2 culture and infection. a Image of the HSV-1 plagues in infected Vero cells after staining with $1 \%$ naphtalene blue. b Changes in THBMEC morphology after infection with HSV-1 at various MOI. c Image of the HSV-2 plagues in infected Vero cells after staining with $1 \%$ naphtalene blue. d Changes in THBMEC morphology after infection with HSV-2 at various MOI.

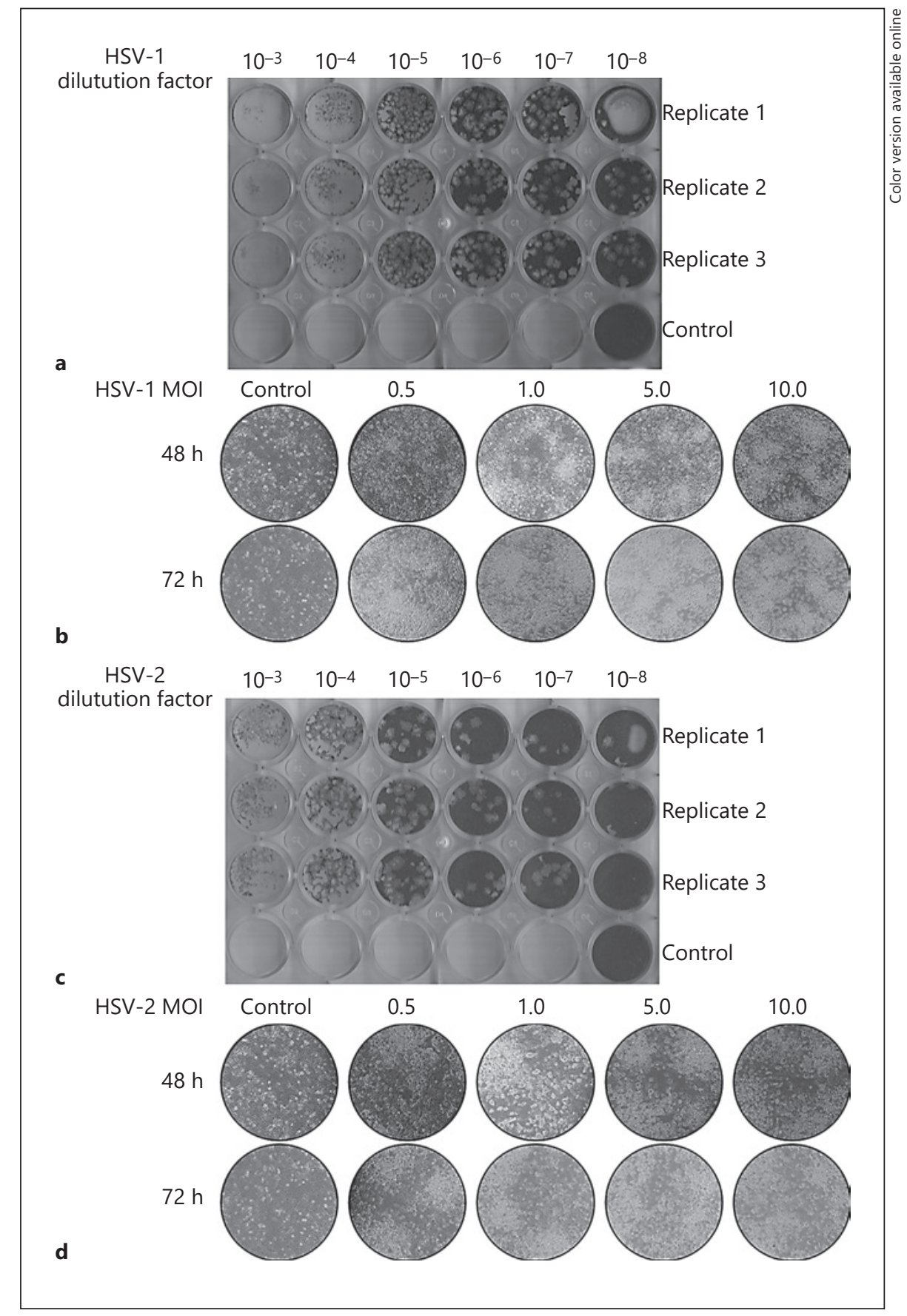

\section{Virus Titration}

The virus titer was determined using a conventional plaque assay and it was expressed as plaque-forming units (pfu) per milliliter. Briefly, the HSV-1 and HSV-2 virus stocks were 10-fold serially diluted in maintenance medium and added to designated wells containing 95\% confluent Vero cells in 24-wells plates (Nunc, Denmark). During the 1-h incubation after infection, the infected cells were occasionally mixed to ensure an even virus distribution.
After approximately $3 \mathrm{~h}$ of virus addition, overlay medium containing 3\% carboxylmethyl cellulose (Calbiochem, USA) with 3\% heat-inactivated FBS in DMEM was added to each well and further incubated for 3-4 days. The cells were checked daily for plaque formation. On the final day, the overlay medium was removed, the cells were rinsed gently with phosphate-buffered saline and stained with $0.1 \%$ naphthalene blue black for visualization, and the number of plaques was counted. 
HSV Infection of Vero and THBMEC

Vero cells were grown in 24-wells plate and infected with HSV1 and HSV-2 at multiplicities of infection (MOI) of 0.5, 1.0, 5.0, and 10.0 to determine the best MOI to be used on THBMEC, the in vitro BBB model. For the ECIS experiments, THBMEC were grown to confluence in 8-well electrode arrays (8W10E+; Applied Biophysics, USA) prior to virus infection. MOI of 0.5 and 5.0 were used in HSV infection. A control group with THBMEC added with non-HSV-infected Vero cell culture supernatant was also included for data normalization purposes.

\section{Real-Time Endothelial Permeability Measurement of}

HSV-Infected THBMEC

ECIS is an experimental technique that monitors cell attachment and spreading in quantitative and real-time manners via measurement of alternating current impedance changes through small gold film electrodes coated on a culture dish as a growth substrate [16]. Using this ECIS tool, we measured the endothelial permeability as a means to evaluate cell-cell barrier function in vitro. The trans-endothelial electrical resistance (TEER) changes when THBMEC were infected with HSV-1 or HSV-2 were recorded at a low frequency $(4 \mathrm{kHz})$ using ECIS $\mathrm{Z} \theta$ with the 8 -well array station (Applied Biophysics). Meanwhile, high-frequency capacitance measurement (at $64 \mathrm{kHz}$ ) was used to monitor the coverage of the electrode surface as the cells were spread. Real-time changes were observed and recorded every $160 \mathrm{~s}$ at multiple frequencies for 5 days after infection. Real-time data were analyzed using ECIS software for Windows (Applied Biophysics). The mathematical model for changes in impedance used to examine cell morphological parameters included cell barrier resistance function $\left(\mathrm{R}_{\mathrm{b}} ; \Omega \bullet \mathrm{cm}^{2}\right)$, cell-matrix resistance function $\left(\alpha ; \Omega^{0.5} \bullet \mathrm{cm}\right)$, and trans-cellular resistance function $\left(\mathrm{C}_{\mathrm{m}} ; \mu \mathrm{F} / \mathrm{cm}^{2}\right)$. Data are also expressed as relative changes of resistance and capacitance values. Each experiment was performed in triplicate and the results are expressed as means \pm SEM.

\section{Results}

\section{THBMEC Was Susceptible to HSV Infection}

Large batches of HSV-1 and HSV-2 were successfully cultured and good titers of the viruses were obtained at $1.6 \times 10^{9}$ (Fig. 1a) and $3.5 \times 10^{8} \mathrm{pfu} / \mathrm{mL}$ (Fig. 1c), respectively. Using these viruses, the susceptibility of the THBMEC was examined by infecting the cells at varying MOI from 0.5 up to 10.0. The results (Fig. 1b, d) show that endothelial cells were infected by the viruses even at MOI as low as 0.5 at $48 \mathrm{~h}$ postinfection (p.i.), as indicated by the formation of multiple multinucleated giant cells (syncytia) in the infected cell monolayer as compared to the control. As the infection MOI and/or the incubation time was increased, the size and number of the formed syncytia also increased.

\section{HSV-1 and -2 Displayed Differential Infectivity in}

THBMEC

The infectivity level of HSV in THBMEC was examined based on the MOI used and the virus types. For MOI comparison, the study parameter used for this analysis was capacitance $(\mathrm{nF})$, which represented the electrical current-insulating capability of the cell monolayer. When the cells were healthy and intact, the capacitance reading was low. However, when the cells lost their intactness as a result of a virus assault, the capacitance reading was increased. This phenomenon is clearly observed in Figure $2 \mathrm{a}$, whereby the normalized capacitance reading after the endothelial cells were infected with HSV-1 at an MOI of 0.5 increased gradually up to $36 \mathrm{~h}$ p.i. and then spiked from $48 \mathrm{~h}$ p.i. onward. The virus infectivity was more apparent when the virus amount was increased to an MOI of 5.0, which became evident at $36 \mathrm{~h}$ p.i. A similar pattern was observed for HSV-2 infection in THBMEC (Fig. 2b), whereby the normalized capacitance reading obtained was increased when a higher MOI of virus and a longer incubation time were applied, with a more profound 2-fold capacitance difference between the 2 MOI tested starting at $48 \mathrm{~h}$ p.i.

Additionally, the infectivity of both HSV-1 and HSV-2 in THBMEC at an MOI of 5.0 was also compared. For this analysis, a resistance parameter which resembled the integrity of the endothelial cell monolayer or barrier function was also included in combination with capacitance measurements. When the tight junctions between cells became weaker due to virus infection, there was less resistance for the electrical current to flow through the cell-cell spaces and hence the reading obtained was decreased. As shown in Figure 2c, HSV-1 (lower capacitance, higher resistance) demonstrated a milder infection in the THBMEC compared to HSV-2 (higher capacitance, lower resistance) until $32 \mathrm{~h}$ p.i. On top of that, the infectivity of HSV-2 in endothelial cells became pronounced much earlier than that of HSV-1, i.e., as early as $8 \mathrm{~h}$ p.i. However, this trend reversed at $32 \mathrm{~h}$ p.i., whereby HSV-1 became much more lethal compared to HSV-2, and this continued until the experiment was terminated at 5 days p.i.

\section{Early HSV Infection Targets the Tight Junction of THBMEC}

Here, we showed for the first time that an acute HSV infection caused early activation of the THBMEC, as infection of endothelial cells with both HSV types resulted in an immediate change in the recorded total resistance data, beginning at $8-15 \mathrm{~h}$ p.i. (Fig. $2 \mathrm{c}, 3$ ). However, in the infected THBMEC up to $10 \mathrm{~h}$ p.i., there was a noticeable 
Fig. 2. Comparison of HSV subtype infections at different MOI in THBMEC. Comparison of infectivity levels for HSV-1 (a) and HSV-2 (b) at MOI of 0.5 and 5.0. c Comparison of infectivity levels for HSV1 and HSV-2 at an MOI of 5.0. Data are expressed as means \pm SEM.
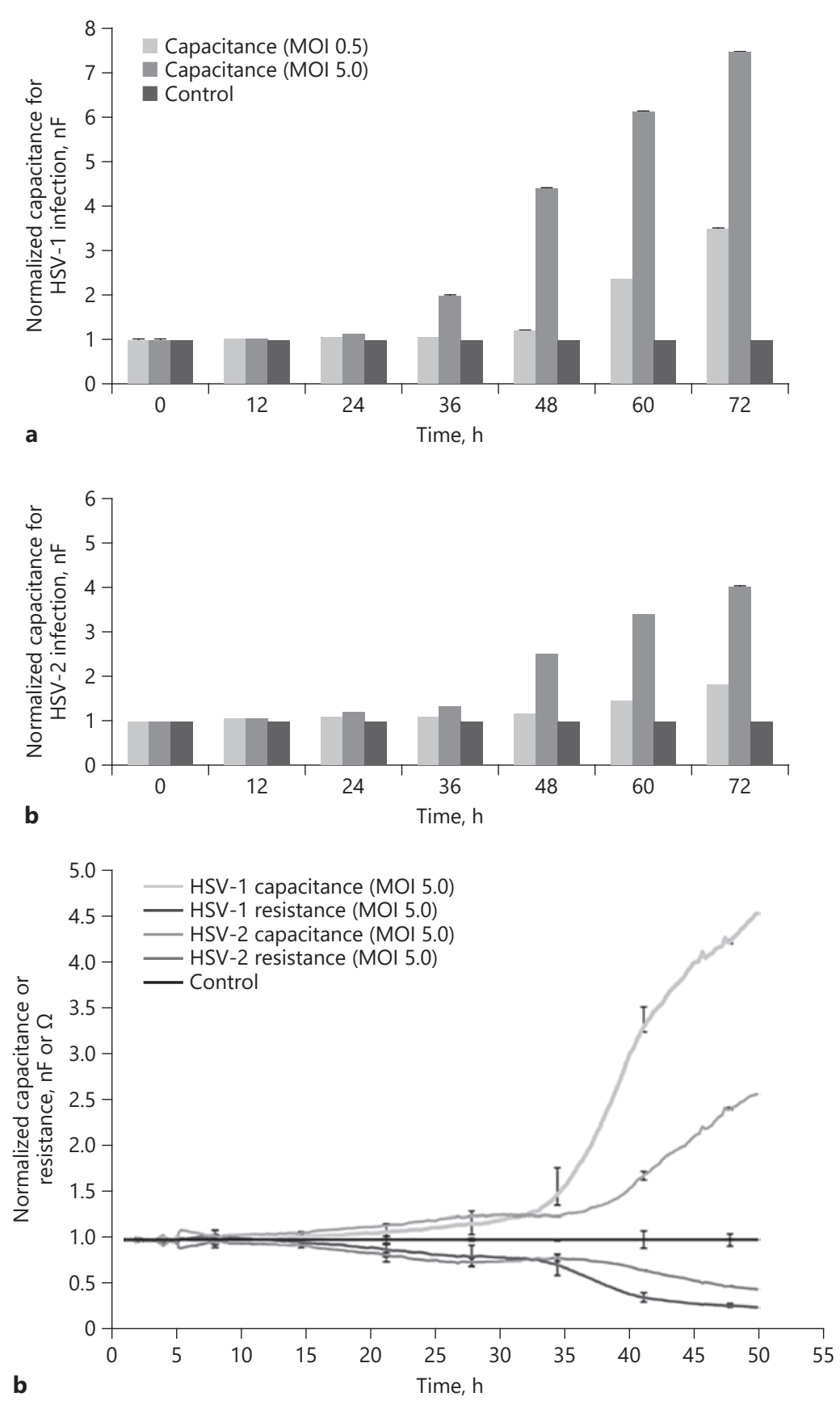

difference between virus infection by HSV-1 and HSV-2 (Fig. 3a). Regardless of the MOI tested, HSV-1 infection seemed to exert an initial brief tightening of the endothelial cell-cell barrier with a slightly higher resistance than the control, while HSV-2 infection caused weakening of the cell-cell junction integrity. Nevertheless, a preceding drop in resistance was generally noted in the HSV-infect- ed cells up to $30 \mathrm{~h}$ p.i. before a change in capacitance was observed (Fig. 3a, b).

Alterations in total resistance could be attributed to the following 2 factors: cell-cell barrier resistance $\left(\mathrm{R}_{\mathrm{b}}\right)$ and cell-matrix resistance $(\alpha)$. Cell-cell barrier resistance implies an obstruction for molecular flow in the intercellular spaces (between cells), whereas cell-matrix resistance im- 
Fig. 3. TEER measurement of THBMEC grown to confluence and after infection with HSV. a Real-time resistance data gathered at $4 \mathrm{kHz}$. b Real-time capacitance data gathered at $64 \mathrm{kHz}$. Data are expressed as means \pm SEM.

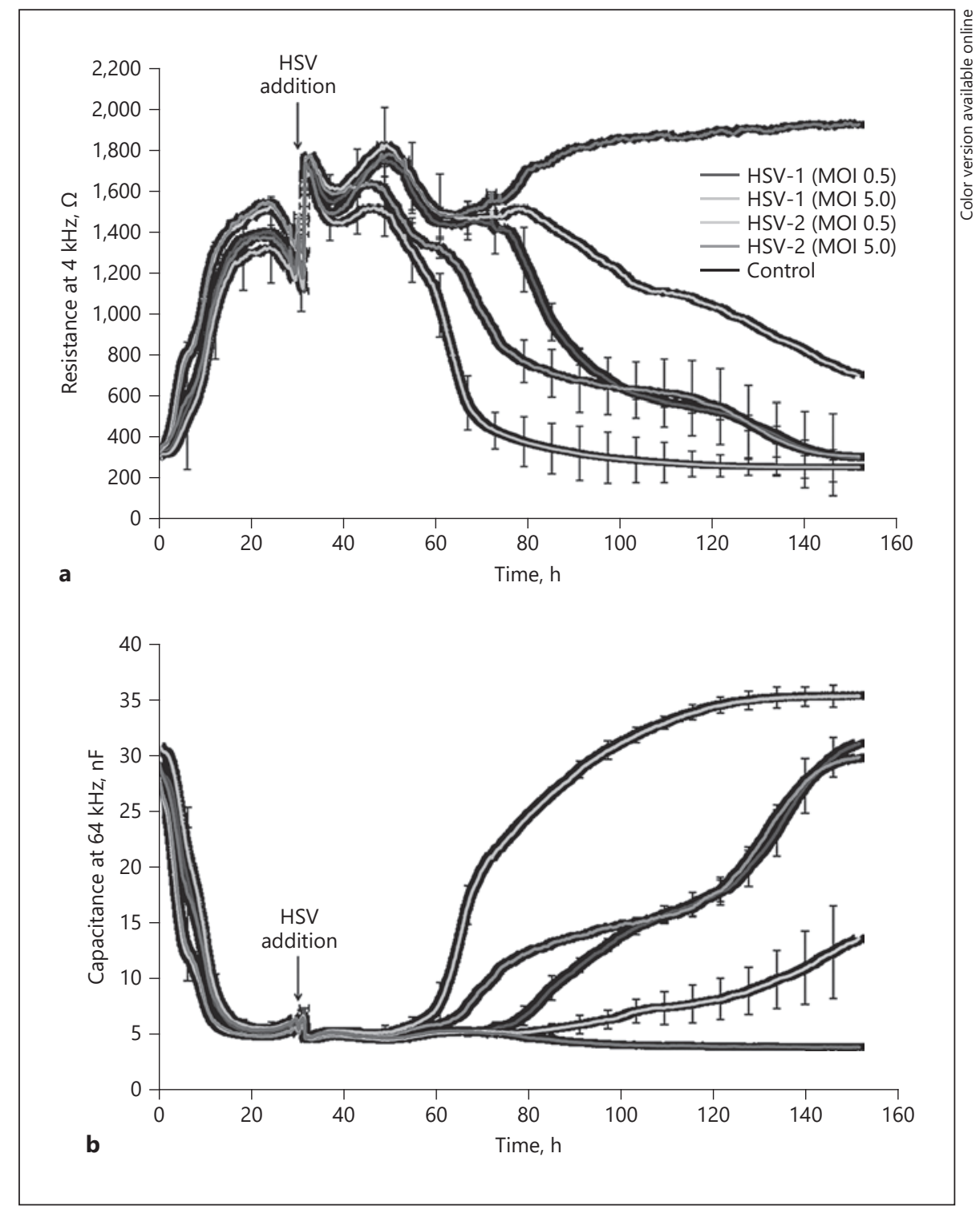

plies blockage of the molecular flow in the pericellular spaces (around cells). Comparing the HSV-1 and HSV-2 infections at an MOI of 5.0 (Fig. 4a, b), there was a decrease in cell-cell barrier resistance at $15 \mathrm{~h}$ p.i., with negligible involvement of cell-matrix adhesion changes. This may indicate that the first immediate target of HSV viruses are the tight junctional proteins between endothelial cells in order to cross the BBB.

Cell Death Was Observed in THBMEC Infected by HSV Mechanistic study of HSV infection in endothelial cells was performed via impedance measurement, which is represented by the 2 most direct parameters, i.e., resis- tance and capacitance. In the case of HSV infection (Fig. 5), we observed an initial gradual decrease in resistance for approximately $30 \mathrm{~h}$ p.i., followed by an increase in capacitance reading. Regardless of the amount of viruses (MOI 0.5 or 5.0 ) added to the endothelial cells, the infection began rather slowly. However, once the HSV infection momentum was reached (at $36 \mathrm{~h}$ p.i.), there were extreme changes in both capacitance and the resistance output, signifying a massive killing of endothelial cells by both the old and the newly replicated viruses. When the infected cells lost their viability, they no longer had the ability to attach to any surface and act as a current-insulating layer, hence leading to a sudden drop in 
Fig. 4. ECIS modeling for THBMEC in response to HSV infection. Graphs illustrating the ECIS modeling parameters normalized against the control. a Barrier resistance function measured by cell-cell adhesion (Rb). b Cell-matrix resistance function measured by cell-substrate adhesion (a). c Trans-cellular resistance function measured by membrane capacitance $\left(\mathrm{C}_{\mathrm{m}}\right)$.
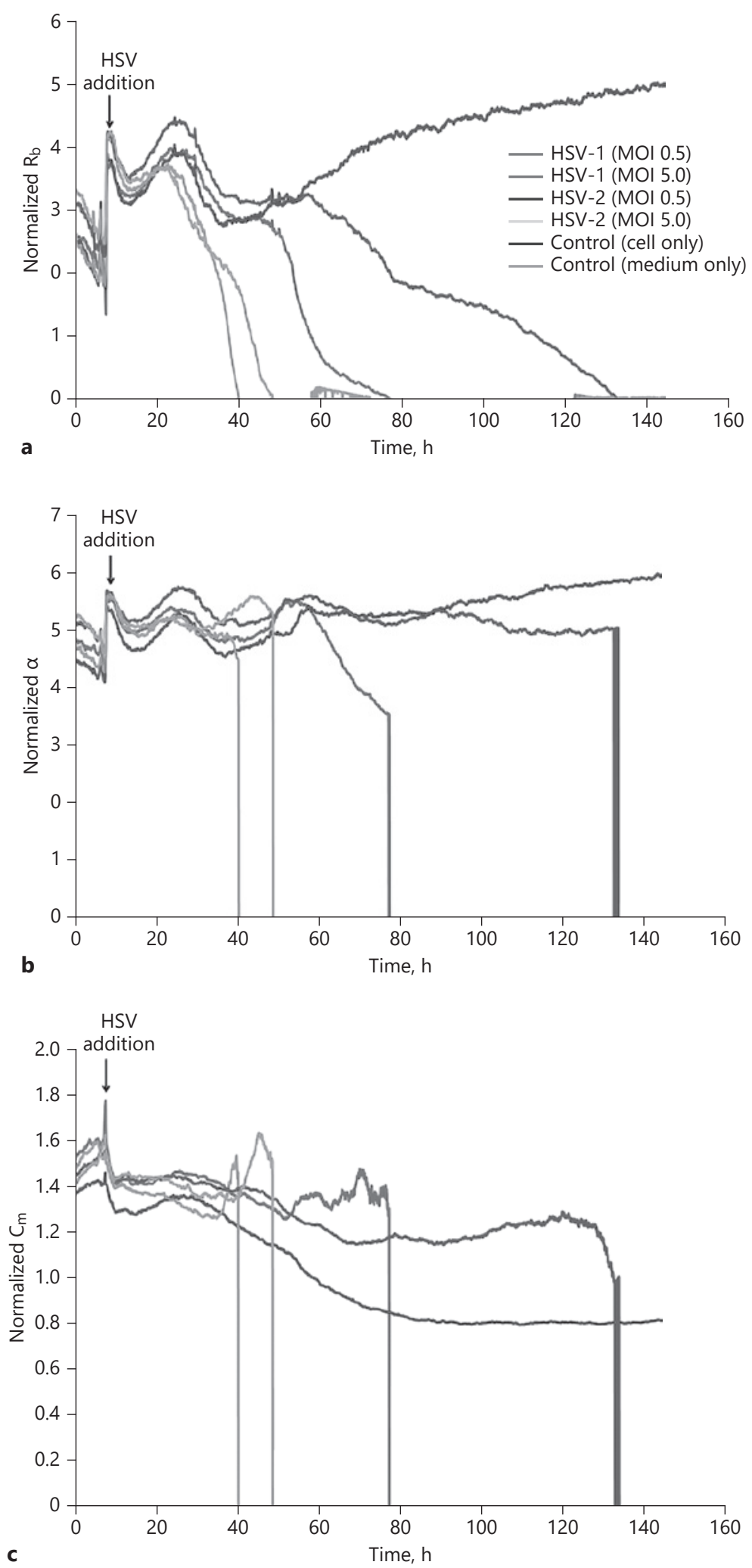
Fig. 5. TEER measurement for THBMEC after infection with HSV. a TEER values shown as normalized resistance in response to the addition of HSV-1 and HSV-2 at MOI of 0.5 and 5.0, wherein the precipitous decrease in TEER indicates a disruption or "leakiness" to the barrier. b TEER values shown as normalized capacitance in response to the addition of HSV-1 and HSV-2 at MOI of 0.5 and 5.0. Data are expressed as means \pm SEM.

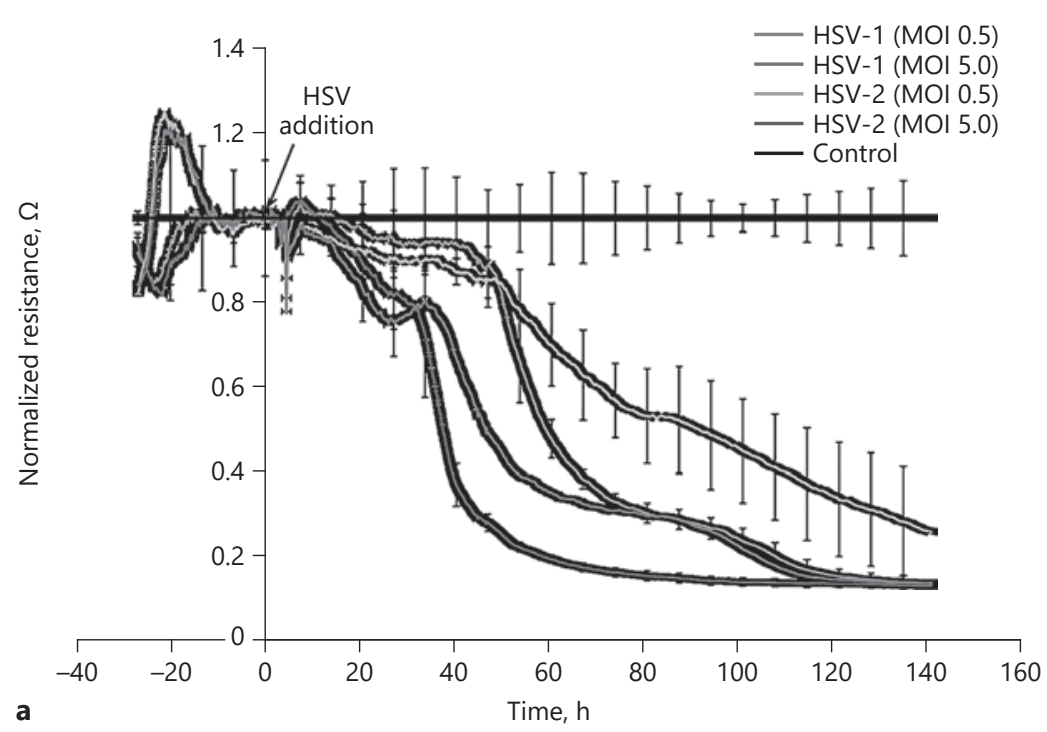

cell-matrix adhesion and membrane capacitance reading that eventually reached the medium only electrode measurement value (Fig. 4).

\section{Discussion}

The human central nervous system is a complex system that controls our body functions by sending instructions or signals primarily via neuronal cells. In order for neurons to work in a highly precise manner, the brain has to be strictly guarded from the external pathogens and microenvironment present in the peripheral blood circulation. For this reason, the BBB exists to distinguish the central nervous system compartment from other body compartments. The lining of endothelium in the BBB differs from the endothelium covering other body organs, as the $\mathrm{BBB}$ endothelium possesses adherent and tight junction proteins that hold them tightly together and, hence, stringently regulate the molecules that can enter and exit the central nervous system [17]. Unfortunately, this tight junction is not impenetrable as viruses or other pathological circumstances could lead to damage or cleavage of these junctional proteins. Depending largely on the viral 
route and the entry site, different neurological symptoms may arise. Some of the common entry mechanisms include lysis of brain cells, apoptosis induction, and hijacking of neuronal cell functions [8].

Among the virus types, herpes viruses are the most common cause of severe neurological morbidity, especially in neonates or immune-compromised individuals [18]. Both HSV-1 and HSV-2 usually transmits through oral mucosa (such as kissing) or genital mucosa (via sexual transmission). Within the stratified squamous epithelium, the transmitted viruses will usually replicate and be transported to the neuronal axon body via a retrograde microtubule-associated process, whereby these viruses will either cause an acute infection or remain latent until a secondary reactivation [19]. In this study, the capability of HSV to infect endothelial cells is clearly shown by the increasing number of syncytia formed as the infection MOI and/or the incubation time were increased. The entire replication cycle of HSV, which ultimately results in destruction of its infected cells, takes approximately 18$20 \mathrm{~h} \mathrm{[20].} \mathrm{This} \mathrm{time} \mathrm{course} \mathrm{fits} \mathrm{perfectly} \mathrm{with} \mathrm{our} \mathrm{HSV-}$ infected THBMEC model whereby a more drastic change in the endothelial cell barrier was observed around 36$48 \mathrm{~h}$ after HSV infection, which is after the second replication cycle of the viruses. At this time point, there could have already been a sufficient virus amount to overwhelm the THBMEC monolayer, leading to a possible breakdown of the BBB protective mechanism. Besides, HSV-1 appeared to be more lethal than HSV-2 based on our findings, although HSV-1 and HSV-2 are two different species with only approximately $50 \%$ genetic differences. One possible explanation could be that primary HSV-2 infection is mostly delayed in the majority of individuals until they reach adolescence or early adulthood, a time where most people would have already been infected by HSV-1 [18]. However, we found that there is no exclusivity in their infection ability in THBMEC as both virus types seem to be able to kill endothelial cells with a high efficiency beginning $18 \mathrm{~h}$ after HSV infection.

To date, there is no report showing a direct correlation between HSV infection and BBB damage in brain encephalitis. However, it is wise to note that the interaction among the virus and its host at the BBB involves very complex mechanisms. There are various ways that a virus could destroy the BBB layer, including the oxidative stress pathway, expression of proteolytic enzymes such as matrix metalloproteinase (MMP), production of inflammatory cytokines, and suppression of tight junction protein expression within the host cell. Alternatively, some viruses could attach themselves to tight junction proteins

HSV-Compromised Brain Endothelial Cell Integrity as their entry mode, resulting in damage to endothelial cells at BBB [2]. Despite the common belief that HSV infects the central nervous system through a neuronal route, there is no evidence so far showing that HSV could not target BBB endothelial cells to cause viral encephalitis. In an HSVE study performed using a mouse model, MMP-9 activity restricted to the brain vasculature area was found to be increased, with a concurrent decrease in the expression of its endogenous inhibitor, hence leading to a decrease in collagen type IV [21]. This extracellular matrix degradation is one possible mechanism for HSV to gain entry into the cerebral region. On top of that, HSV infection has been shown to upregulate mRNA expression of a myriad of cytokines, which include IFN- $\alpha$, IFN- $\beta$, IL28 , IL-29, TNF- $\alpha$, CCL5, and CXCL10. Subsequent protein studies also confirmed a significantly higher induced expression of TNF- $\alpha$, CXCL10, and CCL5 in cells upon infection by HSV [22]. In a separate study, Balb/c mice immunized with a recombinant protein of HSV-1 ectodomain triggered secretion of $\mathrm{T}$ helper cells type 1 (TNF, IFN- $\gamma$, and IL-2) and T helper cells type 2 (IL-4 and -6) cytokines which were detected in the medium fluid of purified peripheral blood and splenocyte cultures [23]. Production of these cytokines could initiate an inflammatory response that facilitates the entry of HSV into the cerebral cortex. Generation of oxidative stress molecules might also be another brain-invading mechanism utilized by HSV as HSV-1 has been shown to be capable of stimulating elevated intracellular reactive oxygen species in infected microglial cell cultures [24] as well as increased bioactive lipid peroxidation by-products generated in infected mouse P19N neural cell cultures [25]. Although the above-mentioned evidence does not directly link HSV infection with $\mathrm{BBB}$ damage, it does point toward the possibility that HSV could indeed affect the BBB using the common mechanisms utilized by other virus types. Our findings from this experiment actually support the hypothesis whereby direct infection of the cultured human brain micro-vascular endothelial cells with either HSV-1 or HSV-2 increased capacitance through the membrane (signifying a breach in the cell membrane) with an initial reduced resistance through the cell-cell barrier (indicating compromised cell-cell contact). Resistance measurement at a lower frequency $(4 \mathrm{kHz})$ reflects blockage of the current flow through the spaces between or underneath cells, while capacitance measured at a higher frequency $(64 \mathrm{kHz})$ is ideal to analyze the capacitive behavior of the cell membrane toward the electrical current. Depending on the infectious nature of the virus, different combinations of resistance and capacitance results could be 
achieved. If a virus invades by breaking through the cellcell barrier without harming the cells themselves, the resistance reading obtained for the infected group will fall below that of the control group, with a consistent capacitance output. On the other hand, if a virus acts by directly attacking the cells and hence collapsing the protective cell-cell barrier, the resistance output from the infected group will drop suddenly, with a concurrent high capacitance reading than that of the control group. These observations and the results obtained show that HSV probably acts via the following mechanisms: (1) destruction of the endothelial cell-cell barrier and (2) killing of endothelial cells, therefore acutely collapsing the endothelial cell monolayer that results in compromised BBB function.

\section{Conclusion}

In summary, more in-depth study of HSV infection is essential for us to further understand severe acute HSVE disease. Through new discoveries and knowledge, we might be able to advance the existing therapies or develop alternative therapies for HSV infection. As a therapeutic modality, this novel discovery could also help us to improvise the use of HSV as a gene-delivery vehicle for other neurological diseases. With this study, we were able to demonstrate for the first time a direct association between HSV infection and destruction of the THBMEC monolayer, a model for BBB functional studies. How- ever, additional detailed investigations are necessary for us to comprehend the actual mechanisms involved and the sequential events of BBB damage caused by HSV invasion.

\section{Acknowledgement}

We would like to thank Dr. Anna Durban for providing us the THBMEC cell line.

\section{Disclosure Statement}

None.

\section{Funding Sources}

This study was supported by a University of Malaya Research Grant (No. RP021C-13HTM).

\section{Author Contributions}

All of the authors contributed equally to this work. S.H.L. and S.D.S. designed this study, performed the experiments, analyzed the data, and wrote and revised this paper; N.A. designed this study and revised this paper; S.M.W. wrote and revised this paper; S.D.S., R.M., and C.S.R. designed this study, analyzed the data, and revised this paper. All of the authors discussed the results and commented on this paper at all stages.

\section{References}

1 Granerod J, Crowcroft NS. The epidemiology of acute encephalitis. Neuropsychol Rehabil. 2007 Aug-Oct;17(4-5):406-28.

2 Spindler KR, Hsu TH. Viral disruption of the blood-brain barrier. Trends Microbiol. 2012 Jun;20(6):282-90.

3 Kanwar JR, Sriramoju B, Kanwar RK. Neurological disorders and therapeutics targeted to surmount the blood-brain barrier. Int J Nanomedicine. 2012;7:3259-78.

4 Dolan A, Jamieson FE, Cunningham C, Barnett $\mathrm{BC}, \mathrm{McGeoch} \mathrm{DJ}$. The genome sequence of herpes simplex virus type 2. J Virol. 1998 Mar;72(3):2010-21.

5 Norberg P, Kasubi MJ, Haarr L, Bergström T, Liljeqvist JA. Divergence and recombination of clinical herpes simplex virus type 2 isolates. J Virol. 2007 Dec;81(23):13158-67.

6 Rigamonti A, Lauria G, Mantero V, Salmaggi A. A case of late herpes simplex encephalitis relapse. J Clin Virol. 2013 Sep;58(1):269-70.
7 Koyuncu OO, Hogue IB, Enquist LW. Virus infections in the nervous system. Cell Host Microbe. 2013 Apr;13(4):379-93.

8 van den Pol AN. Viral infection leading to brain dysfunction: more prevalent than appreciated? Neuron. 2009 Oct;64(1):17-20.

9 Stolwijk JA, Matrougui K, Renken CW, Trebak M. Impedance analysis of GPCR-mediated changes in endothelial barrier function: overview and fundamental considerations for stable and reproducible measurements. Pflugers Arch. 2015 Oct;467(10):2193-218.

10 Szulcek R, Bogaard HJ, van Nieuw Amerongen GP. Electric cell-substrate impedance sensing for the quantification of endothelial proliferation, barrier function, and motility. J Vis Exp. 2014 Mar;(85). https://doi. org $/ 10.3791 / 51300$
11 Man S, Ubogu EE, Williams KA, Tucky B, Callahan MK, Ransohoff RM. Human brain microvascular endothelial cells and umbilical vein endothelial cells differentially facilitate leukocyte recruitment and utilize chemokines for T cell migration. Clin Dev Immunol. 2008; 2008:384982.

12 Yosef N, Ubogu EE. An immortalized human blood-nerve barrier endothelial cell line for in vitro permeability studies. Cell Mol Neurobiol. 2013 Mar;33(2):175-86.

13 Tan WC, Jaganath IB, Manikam R, Sekaran SD. Evaluation of antiviral activities of four local Malaysian Phyllanthus species against herpes simplex viruses and possible antiviral target. Int J Med Sci. 2013 Oct;10(13):181729.

14 Blakeney S, Kowalski J, Tummolo D, DeStefano J, Cooper D, Guo M, et al. Herpes simplex virus type 2 UL24 gene is a virulence determinant in murine and guinea pig disease models. J Virol. 2005 Aug;79(16):10498-506. 
15 Dedera D, Ratner L. Demonstration of two distinct cytopathic effects with syncytium formation-defective human immunodeficiency virus type 1 mutants. J Virol. 1991 Nov; 65(11):6129-36.

16 Wegener J, Keese CR, Giaever I. Electric cellsubstrate impedance sensing (ECIS) as a noninvasive means to monitor the kinetics of cell spreading to artificial surfaces. Exp Cell Res. 2000 Aug;259(1):158-66.

17 Abbott NJ. Blood-brain barrier structure and function and the challenges for CNS drug delivery. J Inherit Metab Dis. 2013 May;36(3): 437-49.

18 Berger JR, Houff S. Neurological complications of herpes simplex virus type 2 infection. Arch Neurol. 2008 May;65(5):596-600.
19 Cunningham AL, Diefenbach RJ, MirandaSaksena M, Bosnjak L, Kim M, Jones C, et al. The cycle of human herpes simplex virus infection: virus transport and immune control. J Infect Dis. 2006 Sep;194(s1 Suppl 1):S11-8.

20 Jacobs A, Breakefield XO, Fraefel C. HSV-1based vectors for gene therapy of neurological diseases and brain tumors: part I. HSV-1 structure, replication and pathogenesis. Neoplasia. 1999 Nov; 1(5):387-401.

21 Sellner J, Simon F, Meyding-Lamade U, Leib SL. Herpes-simplex virus encephalitis is characterized by an early MMP-9 increase and collagen type IV degradation. Brain Res. 2006 Dec;1125(1):155-62.

22 Melchjorsen J, Sirén J, Julkunen I, Paludan SR, Matikainen S. Induction of cytokine expression by herpes simplex virus in human monocyte-derived macrophages and dendritic cells is dependent on virus replication and is counteracted by ICP27 targeting NF-kap$\mathrm{paB}$ and IRF-3. J Gen Virol. 2006 May;87(Pt 5):1099-108.
23 Durmanová V, Sapák M, Kosovský J, Rezuchová I, Kúdelová M, Buc M, et al. Immune response and cytokine production following immunization with experimental herpes simplex virus 1 (HSV-1) vaccines. Folia Microbiol (Praha). 2008;53(1):73-83.

24 Schachtele SJ, Hu S, Little MR, Lokensgard JR. Herpes simplex virus induces neural oxidative damage via microglial cell Toll-like receptor-2. J Neuroinflammation. 2010 Jun;7(1): 35.

25 Kavouras JH, Prandovszky E, Valyi-Nagy K, Kovacs SK, Tiwari V, Kovacs M, et al. Herpes simplex virus type 1 infection induces oxidative stress and the release of bioactive lipid peroxidation by-products in mouse P19N neural cell cultures. J Neurovirol. 2007 Oct; 13(5):416-25. 